a bacterial meningitis than a first seizure, and the common emphasis on routine taps for first seizures with fever may be misleading.

Another source of confusion is the determination of complex features of febrile seizures. Berg AT et al. have found disagreement among pediatric neurologists regarding the assessment of focality of the seizure (Epilepsia 1992; $\underline{33}: 661-666)$. The reasons for disagreement included variation in interpretation of lateral eye deviation, staring episodes and motor asymmetries of the convulsion.

\title{
ANTICONVULSANTS FOLLOWING CRANIOTOMY
}

A randomized prospective clinical trial of carbamazepine or phenytoin for 6 or 24 months including a control no-treatment group is reported in 276 post-craniotomy patients at the Walton Hospital, Liverpool, England. 103 (37\%) patients suffered at least 1 seizure. Of those who developed status epilepticus in the first week after operation, $8 \%$ were treated with carbamazepine, $5 \%$ with phenytoin, and $2 \%$ had no treatment. Serum anticonvulsant levels were not monitored in all patients. In those who were monitored the levels were never in the optimal range, particularly in the phenytoin treated group. Early anticonvulsant treatment did not affect the long-term response of epilepsy to anticonvulsant drugs. The occurrence of seizures within the first post-operative week did not increase the likelihood of late epilepsy. Acute allergic skin rashes occurred in 28 (13\%) patients. The authors concluded that prophylactic anticonvulsants should not be recommended routinely following supratentorial craniotomy. (Foy PM et al. Do prophylactic anticonvulsant drugs alter the pattern of seizures after craniotomy? J Neurol Neurosurg Psychiatry Sept 1992; 55:753-757.) (Correspondence: Dr. P.M. Foy, Mersey Regional Department of Medical and Surgical Neurology, Walter Hospital, Liverpool, England.)

COMMENT. The authors admit that the small and uncertain effect of antiepileptic drug treatment in this study might be related to noncompliance and failure to achieve optimal blood levels. Of those blood levels available a significant proportion of phenytoin levels were suboptimal, and it could be argued that the more rigorous monitoring might have improved results. In contrast, satisfactory carbamazepine levels were achieved and yet, there was no difference in outcome. These findings suggest that phenytoin has a greater prophylactic action than carbamazepine in postcraniotomy seizure control. According to Drs. Wingkun and Awad at the Cleveland Clinic, changes in antiepileptic drugs, dosages or poor compliance may be important factors in postoperative recurrence of seizures (see Ped Neurol Briefs Jan $1992 ; \underline{6}: 8)$. The unusually high incidence of skin rashes associated with anticonvulsant treatment in this study suggests that postcraniotomy patients are more susceptible than non-surgical patients with epilepsy. The respective frequency of skin rash with the two anti-convulsants used in this study was not stated. It would be of interest to know if the antibiotic Cefotaxim was administered to these surgical patients in conjunction with the anticonvulsant, since this combination of drugs is 
known to increase the risk of serious skin reactions (see Ped Neurol Briefs May $1992 ; \underline{5}: 37)$.

\section{SEIZURES AND CEREBRAL CYSTICERCOSIS}

The results of a long-term retrospective study of 240 patients with seizures and cysticercosis of the brain parenchyma are reported from the Instituto Nacional de Neurologio y Neurocirugia Manuel Velasco Suarez de Mexico, Mexico City. Of 118 patients who received cysticidal therapy (albendazole, prasiquantel, or both) for lesions without inflammation on imaging studies, there was an $82 \%$ reduction in the mean number of brain cysts and a $95 \%$ reduction in the mean frequency of seizures. After 3 years of follow-up, $54 \%$ of patients were seizure free. By contrast, the untreated patients averaged 11 seizures per year and none was seizure free. Of 58 patients with inflammation around cysts who did not receive cysticidal medication, there was a $74 \%$ reduction in the frequency of seizures and $31 \%$ became seizure free. After surgical treatment of unmedicated patients there was an $87 \%$ reduction in seizure frequency and $40 \%$ became seizure free. (Vazquez V, Sotelo J. The course of seizures after treatment for cerebral cysticercosis. N Engl J Med Sept 3, 1992; 327:696-701.) (Reprints: Dr. Sotelo, National Institue of Neurology and Neurosurgery, Insurgentes sur 3877, 14269, Mexico City, Mexico.)

COMMENT. The outcome for patients with epilepsy due to neurocysticercosis was better after treatment with cysticidal agents than when the primary disease was left to follow its natural course. There were fewer seizures after medical treatment than after surgical excision of the cyst. In the patients with inflammation around cysts, spontaneous resolution of the cysts occurred frequently but the number of seizures per year in this group was $31 \%$ higher than in those who received medical treatment with cysticidal drugs. Granulomas remaining after the cysts destruction may account for the high incidence of persistent seizures. The patients in this study were adults, but most had suffered from frequent seizures for many years because of persistence of the paracystic epileptic focus.

Children in endemic areas who have seizures related to cysticercosis should be considered for cysticidal therapy at the time of diagnosis. The use of these drugs in children may require further definition.

Cysticercosis of the CNS is rare in the United States, but accounts for $25 \%$ of intracranial tumors in Mexico. Increased intracranial pressure is found in about $75 \%$ of cases (Menkes JH. Textbook of Child Neurology $3 \mathrm{rd}$ Ed. Philadelphia. Lee and Febiger 1981). 